\title{
Non-cooperative Games Involving Type-2 Fuzzy Uncertainty: An Approach
}

\author{
Juan Carlos Figueroa-García ${ }^{1}$, Emanuel Jonathan Medina-Pinzón ${ }^{2}$, \\ and Jannan David Rubio-Espinosa ${ }^{2}$ \\ 1 Universidad Distrital Francisco José de Caldas, Bogotá, Colombia \\ 2 Industrial Engineering Department, \\ Universidad Distrital Francisco José de Caldas, Bogotá, Colombia \\ jcfigueroag@udistrital.edu.co, \\ ejmedinap@gmail.com, darubios@hotmail.com
}

\begin{abstract}
Classical games theory is based on a set of deterministic payoffs that represent different strategies taken by its players. Deterministic (crisp) payoffs are used by homogeneous players while in many social and business scenarios, different uncertainties such as numerical, linguistic, stochastic, etc are involved. This way, we proposed a model for a two-player game problem involving nonprobabilistic uncertainty coming from uncertain fuzzy payoffs, through Interval Type-2 fuzzy numbers. A method for solving this kind of uncertain games is proposed, and an introductory example is shown.
\end{abstract}

\section{Introduction and Motivation}

Game theory became an important discipline, widely applied in fields such as economics, financial, engineering, etc (see Nash [1], and Neumann and Morgenstern [2]). In some cases, uncertainty affects the payoffs of a game, so in real scenarios both the behavior of the payoffs and the strategies taken by players become an important topic in game theory. In classical (crisp) two-person games the payoffs are deterministic numbers, but in some cases the payoffs contain imprecision that can be handled through fuzzy numbers; in this case the game is called a Fuzzy Game.

Fuzzy games in literature are based on the use of classical fuzzy sets (a.k.a Type-1 fuzzy sets) alongside Linear Programming ( $L P)$ methods. The most important works have been proposed by Bector and Chandras [3], Campos [4], Delgado, Verdegay and Vila [5], Li [6], Butnariu [7, 8], Vijay et al [9], Monroy et al [10], Cunlin and Qiang [11], and Larbani [12]. All those works handle imprecision around the payoffs in the game using Type-1 fuzzy sets/numbers.

It is commonly assumed that the payoffs of a game are perfectly defined without uncertainty. In some cases the payoffs cannot be surely defined due to multiple uncertainties, and they are defined by multiple people who express their perceptions about the payoffs of a game. As usual, those perceptions are not deterministic values and they involve multiple aspects that every person believes possible, so different people's thoughts lead to have multiple fuzzy sets. This way, a fuzzy game composed by multiple people defining its payoffs needs special tools to handle this kind of fuzzy uncertainty.

Our proposal is based on the use of Type-2 fuzzy numbers to involve all perceptions and opinions of who define the payoffs of a game, dealing with non-consensus 
and linguistic uncertainty around their perceptions. Using LP methods, the extension principle, and the decomposition theorem, we provide a mathematical framework for solving a matrix game conformed by two-players under uncertainty coming from disagreement and non-consensuses on the definition of the payoffs of the game.

We also use interval Type-2 fuzzy numbers to represent the set of possible solutions that the players may use, given a particular perception about the payoffs. A method based on $\alpha$-cuts to solve such games is proposed and explained through an example.

The paper has seven sections. Section 1 shows the Introduction and Motivation. In Section 2, the classical two-players game and its LP formulation are presented. In Section 3, basics on Type-2 fuzzy numbers are recalled. In Section 4, a Type-2 fuzzy game model is presented. Section 5 presents the proposal for solving the problem; Section 6 shows an application example, and Section presents 7 some concluding remarks.

\section{The Classical Two-Players Game}

In a two-players game, they are trying to make a move to earn the best possible benefits, given possible moves of the other player and known payoffs of every move. In zero-sum games, a move gives a gain to a player while its opponent losses the same. Then a game $G$ is conformed by a set $S^{m}$ of strategies of the Player I and a set $S^{n}$ of strategies of the Player II. If Player II selects the pure strategy $s_{j}$ and the Player I selects the pure strategy $s_{i}$, then Player II has to pay $a_{i j}$ units to Player I.

All payoffs of the game are comprised into a payoff matrix namely $A$, where each element $a_{i j}$ is the payoff of the Player I, and a negative value means that Player I has to pay $a_{i j}$ to Player II, as shown next:

$$
A=\left(\begin{array}{cccccc}
a_{11} & a_{12} & \cdots & a_{1 j} & \cdots & a_{1 n} \\
a_{21} & a_{22} & \cdots & a_{2 j} & \cdots & a_{2 n} \\
\vdots & \vdots & \ddots & \vdots & \cdots & \vdots \\
a_{i 1} & a_{i 2} & \cdots & a_{i j} & \cdots & a_{i n} \\
\vdots & \vdots & \ddots & \vdots & \cdots & \vdots \\
a_{m 1} & a_{m 2} & \cdots & a_{m j} & \cdots & a_{m n}
\end{array}\right)
$$

where $a_{i j} \in \mathbb{R}, i \in\{1,2, \cdots, m\}$ and $j \in\{1,2, \cdots, n\}$.

This matrix $A$ was originally defined with deterministic (crisp) payoffs, which leads to have only one strategy that maximizes the game. Decision making for Player I uses a max - min principle which seeks to maximize its income through the worse possible movings done by its opponent, so the final decision consists in doing a move $i$ that earns the maximum of the minimum payoffs given all possible moves $j$ of its opponent ( $\max -\min$ principle is applied to Player II).

\subsection{LP Formulation}

Basically, an LP formulation of a two-players game is intended to find the optimal frequency of moves $x_{i}^{*}$ which maximizes the value of the game. To do so, Player I has to select a move $x_{i}^{*}\left(S^{m}\right)$ to obtain the best expected payoff $\left(a_{i j}\right)$. 


$$
\begin{gathered}
\operatorname{Max}_{x \in S^{m}}\left\{\operatorname{Min}\left(\sum_{i=1}^{m} a_{i 1} x_{i}, \sum_{i=1}^{m} a_{i 2} x_{i}, \cdots, \sum_{i=1}^{m} a_{i n} x_{i}\right)\right\} \\
\text { s.t. } \\
\sum_{i=1}^{m} x_{i}=1 \\
x_{i} \geqslant 0
\end{gathered}
$$

Dantzig [13] proposed the first LP model for games based on the works of Nash [1], and Neumann \& Morgenstern [2]. His proposal uses an auxiliary variable $v$ that represents the min operator. The LP formulation for Player I is shown next.

$$
\begin{gathered}
\operatorname{Max} z=v \\
\text { s.t. } \\
v-\sum_{i=1}^{m} a_{i j} x_{i} \leqslant 0 \quad \forall j \in S^{n} \\
\sum_{i=1}^{m} x_{i}=1 \\
x_{i} \geqslant 0, \quad v \in \mathbb{R}
\end{gathered}
$$

$v$ is an auxiliary variable which operates as $v=\operatorname{Min}\left(\sum_{i=1}^{m} a_{i 1} x_{i}, \cdots, \sum_{i=1}^{m} a_{i n} x_{i}\right)$.

The LP model for Player II is the dual model of Player I, so the optimal value of the game is the same for both players. Further details can be found in Dantzig [13].

\section{Type-2 Fuzzy Sets}

A Type-2 fuzzy set $\tilde{A}$ (see Mendel [14]) is an ordered pair $\tilde{A}=\left\{\left(x, \mu_{\tilde{A}}(x)\right): x \in X\right\}$, where $A$ is its linguistic label and $u$ represents uncertainty around the word $A$ :

$$
\tilde{A}=\left\{\left((x, u), \mu_{\tilde{A}}(x, u) \mid \forall x \in X, \forall u \in J_{x} \subseteq[0,1]\right\}\right.
$$

where $J_{x} \subseteq[0,1]$ is the set of primary memberships of $\tilde{A}, \mu_{\tilde{A}}(x, u) \in[0,1]$, and $u$ is its domain of uncertainty.

An Interval Type-2 fuzzy set (IT2FS) (see Figure1) is a special kind of T2FS in which $\mu_{\tilde{A}}(x, u)=1$. Hence, an IT2FS $\tilde{A}$ is composed by an infinite amount of Type- 1 fuzzy sets embedded into $J_{x}$. In other words (see Mendel, John \& Liu [15])

$$
\tilde{A}=\left\{\left(x, \mu_{\tilde{A}}(x)\right) \mid x \in X\right\}
$$

where $\mu_{\tilde{A}}(x)$ is completely characterized by $J_{x} \subseteq[0,1]$.

Here, $\tilde{A}$ is an Interval Type- 2 fuzzy set defined over a domain (usually $\mathbb{R}_{+}$), its support $\operatorname{supp}(\tilde{A})$ is enclosed into the interval $a \in\left[A^{L(+)}, A^{R(+)}\right] . \mu_{\tilde{a}}$ has a linear Type-2 fuzzy 


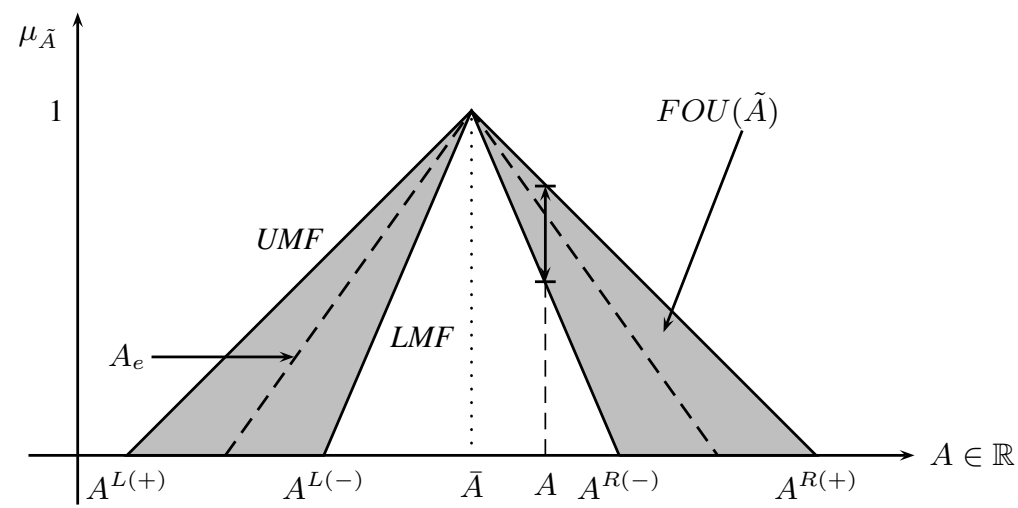

Fig. 1. Interval Type-2 Fuzzy set $\tilde{A}$

set with parameters $A^{L(+)}, A^{L(-)}, A^{R(-)}, A^{R(+)}$ and $\bar{A}$. FOU is the Footprint of Uncertainty of the Type- 2 fuzzy set and $A_{e}$ is an embedded Type-1 fuzzy set into its FOU. Uncertainty $A$ is defined by its FOU as the union of all primary memberships, i.e.

$$
\operatorname{FOU}(\tilde{A})=\bigcup_{x \in X} J_{x}
$$

The FOU of $\tilde{A}$ involves all $J_{x}$ weighted by a secondary membership function $f_{x}(u) /(x, u)$, in this case $1 /(x, u)$. Any FOU is bounded by two membership functions: an Upper membership function $(U M F)=\bar{\mu}_{\tilde{A}}$ and a Lower membership function $(L M F)=\underline{\mu}_{\tilde{A}}$ which have $e$ embedded sets $\left(A_{e}\right)$.

\subsection{The Zadeh's Extension Principle}

The selected approach to to model fuzzy functions is the Zadeh's Extension principle (see Klir and Yuan [16] which is presented as follows

Theorem 3.1. Let $f$ be a function such as $f: X_{1}, X_{2}, \cdots, X_{n} \rightarrow z$, and $A_{i}$ is a fuzzy set in $X_{i}, i=1,2, \cdots, n$ with $x_{i} \in X_{i}$, then we have

$$
f\left(A_{1}, A_{2}, \cdots, A_{n}\right)(z)=\sup _{z=f\left(x_{1}, x_{2}, \cdots, x_{n}\right)} \min _{i}\left\{\mu_{A_{1}}\left(x_{1}\right), \mu_{A_{2}}\left(x_{2}\right), \cdots, \mu_{A_{n}}\left(x_{n}\right)\right\}
$$

Then, we can project any function $z=f\left(x_{1}, x_{2}, \cdots, x_{n}\right)$ into a fuzzy set using their membership functions $\mu_{A_{1}\left(x_{1}\right)}, \mu_{A_{2}\left(x_{2}\right)}, \cdots, \mu_{A_{n}\left(x_{n}\right)}$. For simplicity, we compute the set $\mu_{z}$ using a mapping of $A_{i}\left(x_{i}\right)$ instead of $x_{i}$. This is by using $\alpha$-cuts and the representation theorem of fuzzy set (See Klir and Yuan [17]), presented next.

\subsection{The Extended Representation Theorem}

The $\alpha$-cut of $A$ is defined as ${ }^{\alpha} A=\left\{x \mid \mu_{A}(x) \geqslant \alpha\right\}$. The function $z=f\left(x^{*}\right)$ comes from $f\left(\tilde{A}_{i}\right)(z)$, so $f\left(\tilde{A}_{i}\right)(z)$ can be easily computed through $\alpha$-cuts instead of mapping 
$x_{i}$. Then, we compute a function of $f^{-1}\left(\mu_{\tilde{A}}\right) \rightarrow x$ to finally compute functions of fuzzy sets. The extension of the $\alpha$-cut of $A$ to the $\alpha$-cut of $\tilde{A}$ has been proposed by Figueroa [18] as the union of all possible $\alpha$-cuts over $A_{e},{ }^{\alpha} A_{e}=\left\{x \mid \mu_{A_{e}}(x) \geqslant \alpha\right\}$ :

$$
{ }^{\alpha} \tilde{A}=\left\{(x, u) \mid J_{x} \geqslant \alpha\right\} u \in J_{x} \subseteq[0,1], \alpha \in[0,1]
$$

Now, the boundaries of each $\alpha$-cut are defined as follows (see Figure 2):

$$
\begin{aligned}
{ }^{\alpha} \tilde{A}_{i j}^{L} & =\left[{ }^{\alpha} \tilde{A}_{i j}^{L(+)},{ }^{\alpha} \tilde{A}_{i j}^{L(-)}\right] \\
{ }^{\alpha} \tilde{A}_{i j}^{R} & =\left[{ }^{\alpha} \tilde{A}_{i j}^{R(-)},{ }^{\alpha} \tilde{A}_{i j}^{R(+)}\right]
\end{aligned}
$$

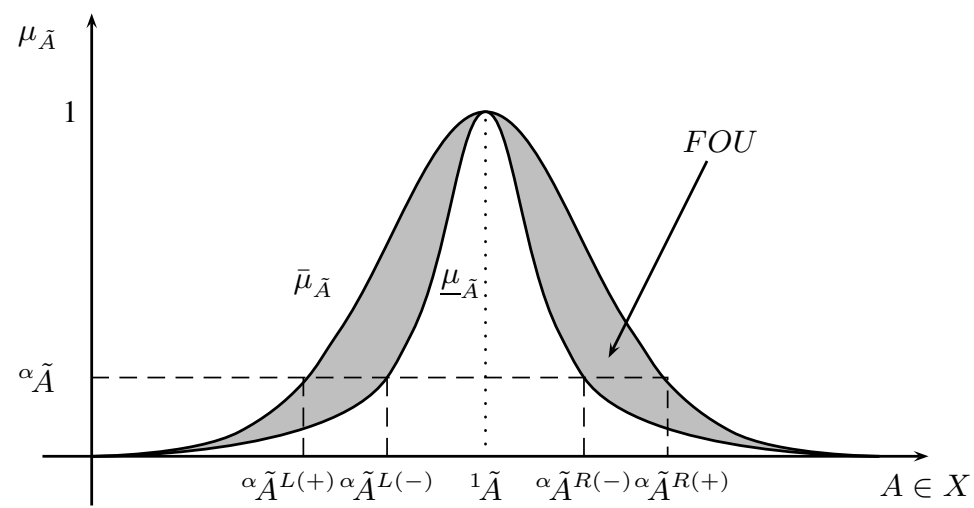

Fig. 2. Fuzzy set $\tilde{A}$

Using (4), we can extend $z$ to a Type-2 fuzzy environment, as follows:

$$
f(\tilde{A})(z)=f^{\alpha}\left(\tilde{A}_{1}, \tilde{A}_{2}, \cdots, \tilde{A}_{n}\right)(z)=f\left({ }^{\alpha} \tilde{A}_{1}\left(x_{1}\right),{ }^{\alpha} \tilde{A}_{2}\left(x_{2}\right), \cdots,{ }^{\alpha} \tilde{A}_{n}\left(x_{n}\right)\right)(z)
$$

\subsection{Type-2 Fuzzy Numbers (IT2FN)}

A Type-2 fuzzy number $(T 2 F N)$ can be seen as the extension of a Type-1 fuzzy number. Then $\tilde{A}$ is a T2FS whose UMF and LMF are normalized fuzzy numbers, ${ }^{\alpha} A$ is a closed interval for all $\alpha \in[0,1]$, and bounded support $\operatorname{supp}(A)$. Now, if both the UMF and LMF of $\tilde{A}$ are fuzzy numbers, then $\tilde{A}$ is an IT2FN. Figure 1 depicts a Type- 2 fuzzy number since both its UMF and LMF are fuzzy numbers as well.

In some applications, a crisp measure (seen either as the expected or most possible value) of an IT2FS, is required. The computation of the expected value of an IT2FN is possible using a centroid-based method, whose details can be found in Figueroa [18], Karnik \& Mendel [19], and Melgarejo [20]. 


\section{The IT2FS Games Model}

We have based our proposal on the Li's $\alpha$-cuts method for fuzzy games (see Li [6]). In our paper, the payoffs matrix has been defined using Interval Type- 2 fuzzy uncertainty, so the problem (in terms of Player I) is as follows:

$$
\begin{gathered}
\operatorname{Max}_{x \in S^{m}}\left\{\operatorname{Min}\left(\sum_{i=1}^{m} \tilde{a}_{i 1} x_{i}, \sum_{i=1}^{m} \tilde{a}_{i 2} x_{i}, \cdots, \sum_{i=1}^{m} \tilde{a}_{i n} x_{i}\right)\right\} \\
\text { s.t. } \\
\sum_{i=1}^{m} x_{i}=1 \\
x_{i} \geqslant 0
\end{gathered}
$$

In this model each $\tilde{a}_{i j}$ is defined as an IT2FN, so its mathematical programming form (for Player I), is:

$$
\begin{gathered}
\operatorname{Max} z=\tilde{v} \\
\text { s.t. } \\
\tilde{v}-\sum_{i=1}^{m} \tilde{a}_{i j} x_{i} \precsim 0 \forall j \in S^{n} \\
\sum_{i=1}^{m} x_{i}=1 \\
x_{i} \geqslant 0
\end{gathered}
$$

Every ${ }^{\alpha} \tilde{A}$ leads to four optimal strategies per cut. So every boundary of ${ }^{\alpha} \tilde{A}$ reaches an optimal value namely ${ }^{\alpha} \tilde{Z}^{L(+)}, \alpha \tilde{Z}^{L(-)},{ }^{\alpha} \tilde{Z}^{R(-)}$ and ${ }^{\alpha} \tilde{Z}^{R(+)}$ :

$$
\begin{aligned}
& { }^{\alpha} \tilde{A}^{L(+)} \rightarrow{ }^{\alpha} \tilde{Z}^{L(+)} \\
& { }^{\alpha} \tilde{A}^{L(-)} \rightarrow{ }^{\alpha} \tilde{Z}^{L(-)} \\
& { }^{\alpha} \tilde{A}^{R(-)} \rightarrow{ }^{\alpha} \tilde{Z}^{R(-)} \\
& { }^{\alpha} \tilde{A}^{R(+)} \rightarrow{ }^{\alpha} \tilde{Z}^{R(+)}
\end{aligned}
$$

After computing all values of the game regarding $\alpha$ then the fuzzy set of optimal solutions namely $\tilde{Z}$ is composed by using $f^{-1}\left({ }^{\alpha} \tilde{A}\right)(z)$.

\section{Solving IT2FGs}

The problem of solving IT2FLPs has been investigated by Figueroa [18, 21 -23], so the optimization of IT2FGs become a natural extension of his results. Note that we face a problem of having infinite Type- 1 fuzzy sets into the FOU of $\tilde{A}$, so we have to deal with the FOU of $\tilde{a}_{i j}$ instead to solve infinite fuzzy LPs.

Now, the proposed method to compute a solution of IT2FG modeled as an LP when having a Type-2 fuzzy payoff matrix is described as follows: 


\section{Proposed $\alpha$-cut Based Method}

1. Select $k \in \mathbb{N}$ amount of $\alpha$-cuts

2. Compute all the $\alpha$-cuts of $\tilde{A}$

3. Solve the $4 k$ LP related problems regarding (12), (13), (14) and (15)

4. Compose $f(\tilde{A})(z)$ using equation (7)

5. Compose $\tilde{Z}$ using $f^{-1}\left({ }^{\alpha} \tilde{A}\right)(z)$

6. Compute $C(\tilde{Z}), z_{l}$ and $z_{u}$ using Figueroa [18]

7. Return $\left[z_{l}, z_{u}\right]$ as the interval of expected values of the game

8. Defuzzify $\tilde{Z}$ using $c_{z}=\left(z_{l}+z_{u}\right) / 2$

\subsection{Decision Making}

Fuzzy Decision making is not based on having a single (global) optimum of the problem, they are based on the idea of having a set of optimal solutions coming from fuzzy information, so our problem needs to compute a set of possible solutions of the game called $\tilde{Z}$, as a function of the initial fuzzy payoffs $\tilde{a}_{i j}$.

Every particular value of ${ }^{\alpha} \tilde{a}_{i j}$ leads to a particular value of the game $z^{*}$ which is satisfactory in different degrees $J_{z} \in\left[\underline{\mu}_{\tilde{Z}}, \bar{\mu}_{\tilde{Z}}\right]$, so the idea is to cover as much as possible scenarios. The centroid of $\tilde{Z}$ provides an idea of what an expectation of the game is, so the analyst can use it to have an idea of the expected value of the game.

Finally, what it is proposed is a map of all possible values of $a_{i j}$ in order to provide the analyst a better idea of the behavior of the fuzzy game.

\section{Application Example}

The example is based on a classical example introduced by Lieberman [24]. The original example is a crisp $4 \times 5$ two-players zero-sum game, where the solution is easily computed using an LP model. Now suppose there is no consensus about the payoffs of the game, so different uncertainties affect its definition (i.e ambiguity, multiple people's opinions, imprecision, etc).

The analyst only has crisp payoffs, but they are not reliable. This way, some experts of the game are asked for their opinions about its payoffs, so we have to comprise all the information given by the experts using Type-2 fuzzy numbers which collects linguistic uncertainty (coming from experts opinions and perceptions) around $a_{i j}$ to finally provide a solution. The initial crisp payoffs are shown next:

Table 1. Crisp payoffs of the example

\begin{tabular}{c|c|c|c|c|c}
\hline$(\mathrm{i}, \mathrm{j})$ & $j=1$ & $j=2$ & $j=3$ & $j=4$ & $j=5$ \\
\hline$i=1$ & 1 & -3 & 2 & -2 & 1 \\
$i=2$ & 2 & 3 & 0 & 3 & -2 \\
$i=3$ & 0 & 4 & -1 & -3 & -2 \\
$i=4$ & 4 & 0 & -2 & 2 & -1 \\
\hline
\end{tabular}


For simplicity, experts were asked to express their opinion around the crisp payoffs shown in Table 1 using the statement "What is your opinion about optimistic and pessimistic payoffs of the strategy $(i, j)$ ?". The words Optimistic and Pessimistic are used to compose triangular fuzzy numbers (namely $T(a, b, c)$ ) for every UMF and LMF. Note that $a$ means pessimistic, $b$ means crisp (see Table 1), and $c$ means optimistic payoff. The collected IT2FNs are presented in Table 2

Table 2. Parameters of the example

\begin{tabular}{c|c|c|c|c|c}
\hline \multicolumn{5}{c}{ Lower membership functions (UMF) of the example } \\
\hline (i,j) & $j=1$ & $j=2$ & $j=3$ & $j=4$ & $j=5$ \\
\hline$i=1$ & $\mathrm{~T}(0.7,1,1.5)$ & $\mathrm{T}(-4,-3,2)$ & $\mathrm{T}(1,2,5)$ & $\mathrm{T}(-2.5,-2,0)$ & $\mathrm{T}(0.8,1,5)$ \\
$i=2$ & $\mathrm{~T}(1.5,2,3)$ & $\mathrm{T}(1.5,3,5)$ & $\mathrm{T}(-1,0,2)$ & $\mathrm{T}(-1,3,4)$ & $\mathrm{T}(-3,-2,1.5)$ \\
$i=3$ & $\mathrm{~T}(-1,0,5)$ & $\mathrm{T}(3,4,6)$ & $\mathrm{T}(-3,-1,1.5)$ & $\mathrm{T}(-4,-3,1)$ & $\mathrm{T}(-3.5,-2,3.5$ \\
$i=4$ & $\mathrm{~T}(-5.5,-4-0)$ & $\mathrm{T}(-0.5,0,1)$ & $\mathrm{T}(-4,-2,1.5)$ & $\mathrm{T}(0,2,3)$ & $\mathrm{T}(-2.5,-1,0.5)$ \\
\hline \multicolumn{5}{c}{ Upper membership functions (UMF) of the example } \\
\hline (i,j) & $j=1$ & $j=2$ & $j=3$ & $j=4$ & $j=5$ \\
\hline$i=1$ & $\mathrm{~T}(0,1,2)$ & $\mathrm{T}(-5,-3,3)$ & $\mathrm{T}(0,2,6)$ & $\mathrm{T}(-3,-2,2)$ & $\mathrm{T}(0.5,1,6)$ \\
$i=2$ & $\mathrm{~T}(0.5,2,4)$ & $\mathrm{T}(1,3,7)$ & $\mathrm{T}(-2,0,3)$ & $\mathrm{T}(-3,3,5)$ & $\mathrm{T}(-5,-2,2)$ \\
$i=3$ & $\mathrm{~T}(-2,0,6)$ & $\mathrm{T}(, 1,4,7)$ & $\mathrm{T}(-5,-1,2)$ & $\mathrm{T}(-5,-3,3)$ & $\mathrm{T}(-4,-2,5)$ \\
$i=4$ & $\mathrm{~T}(-6,-4,2)$ & $\mathrm{T}(-1,0,3)$ & $\mathrm{T}(-6,-2,2)$ & $\mathrm{T}(-2,2,5)$ & $\mathrm{T}(-4,-1,1.5)$ \\
\hline
\end{tabular}

Our example uses the values shown in Table 2 to compose the matrix $\tilde{A}$ whose elements are $\mu_{\tilde{a}_{i j}}$. Now, the game is solved using the $\alpha$-cuts decomposition and the representation theorem.

\subsection{Computational Results}

We have selected $k=21$ equally distributed $\alpha$-cuts and computed ${ }^{\alpha} \tilde{A}$ using (5) and (6). After solving the $4 k$ LP problems related to (1) and (2) (this is, an LP for (12), (13), (14), and (15), we compose $f(\tilde{A})(z)$ using step 3 ) and equation (7). The set of optimal solutions $\tilde{Z}$ is shown in Figure 3 .

A clearer clear idea of the behavior of the game is given by the centroid of $\tilde{Z}$, so we compute $z_{l}$ and $z_{u}$ using Figueroa [18], whosse results are shown next:

$$
C(\tilde{Z}) \in\left[z_{l}, z_{u}\right]=[-0.17637,0.47788] \quad \rightarrow \quad c_{z}=0.15075
$$

Now, $C(\tilde{Z})$ is expected interval of values that both groups of players can expect. Different strategies can be taken in the game, for which we provide a set of possible solutions $x_{i}^{*}$ and $y_{j}^{*}$ (Player I and II respectively) that fits into $C(\tilde{Z})$. This is, a set of solutions whose values $z^{*}$ fit into $C(\tilde{Z})$ having different membership degrees. Note that as more $\alpha$-cuts are computed, we have more strategies into $C(\tilde{Z})$.

\subsection{Discussion of the Results}

We have obtained different solutions coming from different payoffs. For instance, the value $z^{*}=0.427$ is within $C(\tilde{Z})$ and comes from ${ }^{0.8} \tilde{A}^{L(-)}$. The optimal strategy 


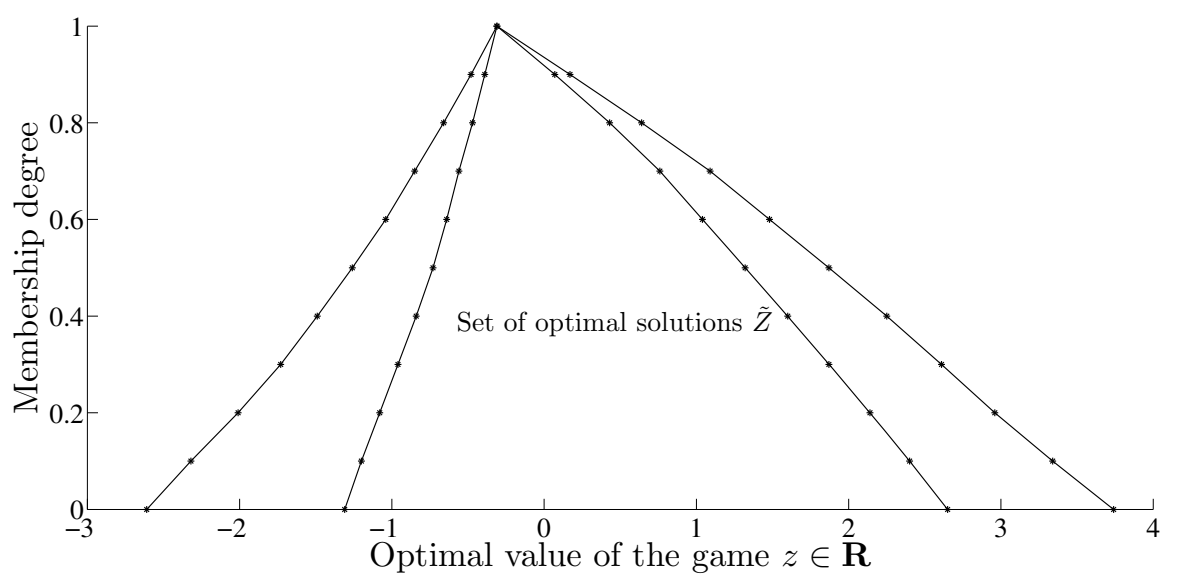

Fig. 3. Set of optimal values of the game $\tilde{Z}$

(probabilities) for Player I is $x^{*}=[0.554,0.425,0.021,0]$, and the optimal strategy for Player II is $y^{*}=[0,0.274,0,0.097,0.629]$. Other example comes from ${ }^{1} \tilde{A}^{R(+)}$ which is equivalent to the crisp solution of the game (outside $C(\tilde{Z})$ ); its optimal value is $z^{*}=-0.306$, the optimal strategy for Player $\mathrm{I}$ is $x^{*}=[0.565,0.354,0.081,0]$, and the optimal strategy for Player II is $y^{*}=[0,0.291,0,0.048,0.661]$.

Other choice is to select a particular payoff. Suppose that players select a payoff, for instance ${ }^{0.8} \tilde{A}^{L(-)}$. This selection leads to $z^{*}=0.427$ whose set of memberships is $\bar{\mu}_{\tilde{Z}}=0.8$ and $\underline{\mu}_{\tilde{Z}} \approx 0.9057$. Note that the value of $\bar{\mu}_{\tilde{Z}}$ is the solution of the game for ${ }_{0.8} \tilde{A}^{R(-)}$, and the value $\underline{\mu}_{\tilde{Z}}$ is computed using the Type-reduction algorithm proposed by Figueroa [18], so the solution satisfies both groups of players in the interval $\alpha \in$ $[0.8,0.9057]$.

\section{Concluding Remarks}

We have presented a method to solve games involving Type-2 fuzzy uncertainty, using the results presented by $\mathrm{Li}$ [6] combined with IT2FNs. It is possible to deal with this kind of uncertainty using well known algorithms, so its applicability to real scenarios is promissory.

The $\alpha$-cuts approach is a map of the optimal solutions of the IT2FG, so as more complete the map of $\tilde{A}$ is, the more complete $\tilde{Z}$ is, giving more information to the analyst. Although there is no a single solution of the IT2FG, we provide a map of the possible strategies that players can take in different scenarios, which is important in decision making under uncertainty.

The use of natural language in optimization and decision making has opened a door to use hybrid methodologies such as the presented in this paper. Different approaches can be proposed to solve this kind of games, so our proposal is just a method for modeling and solving the game. 


\section{References}

1. Nash, J.: Non cooperative games. Annals of Mathematics 54, 286-295 (1951)

2. Neumann, J.V., Morgenstern, O.: Theory of Games and Economic Behavior. Princeton University Press (1944)

3. Bector, C.R., Chandra, S.: Fuzzy Mathematical Programming and Fuzzy Matrix Games. Springer (2005)

4. Campos, L.: Fuzzy linear programming models to solve fuzzy matrix games. Fuzzy Sets and Systems 326(1), 275-289 (1989)

5. Delgado, M., Verdegay, J., Vila, M.: Playing Matrix Games Defined by Linguistic Labels. In: Multiperson Decision Making Models Using Fuzzy Sets and Possibility Theory, pp. 298-310. Springer (1990)

6. Li, D.F.: An effective methodology for solving matrix games with fuzzy payoffs. IEEE Transactions on Cybernetics 43(2), 610-621 (2013)

7. Butnariu, D.: Fuzzy games a description of the concept. Fuzzy Sets and Systems 1, 181-192 (1978)

8. Butnariu, D.: Solution concept for n-Person games. In: Advances in Fuzzy Set Theory and Application, vol. 1. North-Holland Publishing (1979)

9. Vijay, V., Chandra, S., Bector, C.: Bimatrix games with fuzzy payoffs and fuzzy goals. Fuzzy Optimization and Decision Making 3, 327-344 (2004)

10. Monroy, L., Hinojosa, M., Mármol, A., Fernandez, F.: Set-valued cooperative games with fuzzy payoffs. the fuzzy assignment game. European Journal of Operational Research 225(1), 85-90 (2013)

11. Cunlin, L., Qiang, Z.: Nash equilibrium strategy for fuzzy non-cooperative games. Fuzzy Sets and Systems 176(1), 46-55 (2011)

12. Larbani, M.: Non cooperative fuzzy games in normal form: A survey. Fuzzy Sets and Systems 160, 3184-3210 (2009)

13. Dantzig, G.: Linear Programming and Extensions. Princeton University Press (1963)

14. Mendel, J.M.: Uncertain Rule-Based Fuzzy Logic Systems: Introduction and New Directions. Prentice Hall (2001)

15. Mendel, J.M., John, R.I., Liu, F.: Interval type-2 fuzzy logic systems made simple. IEEE Transactions on Fuzzy Systems 14(6), 808-821 (2006)

16. Klir, G.J., Folger, T.A.: Fuzzy Sets, Uncertainty and Information. Prentice Hall (1992)

17. Klir, G.J., Yuan, B.: Fuzzy Sets and Fuzzy Logic: Theory and Applications. Prentice Hall (1995)

18. Figueroa-García, J.C.: An approximation method for type reduction of an interval Type-2 fuzzy set based on $\alpha$-cuts. In: IEEE (ed.) Proceedings of FEDCSIS 2012, pp. 1-6. IEEE (2012)

19. Karnik, N.N., Mendel, J.M.: Centroid of a type-2 fuzzy set. Information Sciences 132(1), 195-220 (2001)

20. Duran, K., Bernal, H., Melgarejo, M.: Improved iterative algorithm for computing the generalized centroid of an interval type-2 fuzzy set. In: 2008 Annual Meeting of the IEEE North American Fuzzy Information Processing Society (NAFIPS) (2008)

21. Figueroa, J.C.: A general model for linear programming with interval type-2 fuzzy technological coefficients. In: 2012 Annual Meeting of the North American Fuzzy Information Processing Society (NAFIPS), pp. 1-6. IEEE (2012)

22. Figueroa-García, J.C., Hernandez, G.: Computing optimal solutions of a linear programming problem with interval type-2 fuzzy constraints. In: Corchado, E., Snášel, V., Abraham, A., Woźniak, M., Graña, M., Cho, S.-B. (eds.) HAIS 2012, Part III. LNCS, vol. 7208, pp. 567576. Springer, Heidelberg (2012)

23. Figueroa-García, J.C., Hernández, G.: A transportation model with interval type-2 fuzzy demands and supplies. In: Huang, D.-S., Jiang, C., Bevilacqua, V., Figueroa, J.C. (eds.) ICIC 2012. LNCS, vol. 7389, pp. 610-617. Springer, Heidelberg (2012)

24. Hillier, F.S., Lieberman, G.J.: Introduction to Operations Research. McGraw-Hill Science (2012) 